\title{
Central facial paresis and its impact on mimicry, psyche and quality of life in patients after stroke
}

\author{
Petr Konecnya ${ }^{\mathrm{a}, \mathrm{b}, \mathrm{c}}$, Milan Elfmark ${ }^{\mathrm{b}, \mathrm{d}}$, Stanislav Horak ${ }^{\mathrm{a}, \mathrm{b}}$, Dalibor Pastucha ${ }^{\mathrm{a}}$, Alois Krobot ${ }^{\mathrm{a}, \mathrm{b}}$, Karel Urbanek ${ }^{\mathrm{c}}$, Petr Kanovsky ${ }^{\mathrm{c}}$
}

Aim. Using functional scales and face video analysis, changes in central facial paresis are monitored in patients with stroke after orofacial therapy and correlations between changes in mimicry, mental function and overall quality of life of patients after stroke are made.

Materials and Methods. A prospective randomized study of patients after stroke with facial paresis. The functional status of the experimental group of 50 cases with orofacial regulation therapy and 49 control cases without mimicry therapy is observed after four weeks of rehabilitation.

Results. Changes in mimicry functions evaluated by the House-Brackmann Grading System (HBGS) clinical range and using 2D video analysis of the distance between the paretic corner of the mouth and earlobe at rest and during smiling were statistically better in the experimental group than in controls. Changes in mental function - depression using Beck Depression Inventory and changes in the quality of life using Bartle index and modified Rankin score (scale) were significantly greater in the experimental group. There was a very close relationship between the changes in mimicry, mental state and overall quality of life according to the Spearman correlative coefficient.

Conclusion. Orofacial rehabilitation therapy for patients with paresis after stroke has a significant influence on the adjustment of mimicry, mental functions and overall quality of life after 4 weeks of treatment.

Key words: stroke, facial paresis, depression, quality of life, orofacial rehabilitation

Received: November 2, 2011; Accepted with revision: February 14, 2013; Available online: March 21, 2013 http://dx.doi.org/10.5507/bp.2013.014

aDepartment of Rehabilitation and Exercise Medicine, Faculty of Medicine and Dentistry, Palacky University Olomouc and University
Hospital Olomouc, Czech Republic
'Department of Physiotherapy, Faculty of Health Sciences, Palacky University Olomouc
'Department of Neurology, Faculty of Medicine and Dentistry, Palacky University Olomouc and University Hospital Olomouc
'Department of Biomechanics and Engineering Cybernetics, Faculty of Physical Culture, Palacky University Olomouc
Corresponding author: Petr Konecny, e-mail:Pet.Konecny@centrum.cz

\section{INTRODUCTION}

One of the most common disorders in patients after stroke is facial paresis (n. VII), which is reflected in a change in mimicry. Stroke is defined as rapidly developing focal or global symptoms of brain function disorder which last more than $24 \mathrm{~h}$ or result in death of the patient, all without other apparent cause than vascular origin ${ }^{1}$.

Mimicry is one of the functions of human communication system. It serves not only to maintain the facial expression but has an important role in speech, in singing, in social communication interaction, in food intake and in eye protection.

Mimicry muscles, which can alter the facial surface in many different ways, execute this function. In addition to opening and closing the eyes and mouth they also have a reporting function. Highly differentiated and complex facial musculature can express a large number of sensations, and can reflect the state of mind and the mood of an individual.

Paresis of the facial nerve after stroke causes a functional and aesthetic defect in a patient which is manifested by facial asymmetry with mimicry muscle disability of the lower half of the face, falling of the corner of the mouth, saliva leakage from the corner of the mouth, asymmetrical smile and speech explicitness disorder with atonia of the lips, tongue and throat ${ }^{1,2}$.

This mimic disability can be qualitatively and quantitatively evaluated. Neurological facial examination, focusing on the examination of the function of the facial nerve, is the principle. On the basis of clinical examination we can diagnose central or peripheral paresis of the facial nerve. We use standard international clinical scales in the quantitative n. VII paresis (paresis of nervus facialis) evaluation. The most used test battery is testing by means of the House-Brackmann Grading System ${ }^{3-5}$.

Mimicry reflects to us, among other things, the psychological state of the patient, and contrarily, mimicry failure may have psychological consequences. Many patients suffer from anxiety and depression after stroke. Within 3 months after stroke $50-60 \%$ of patients suffer from depression ${ }^{6-9}$. Patients with depression after stroke have a worse prognosis. Deterioration in the quality of life is demonstrated with increasing motor movement disability in normal daily activities. Patients have worse rehabilitative care effect, a longer period of hospitalization and are less often able to return to the home environment ${ }^{6,8,10}$. Beck Depression Inventory (BDI-II) test, which quantitatively evaluates the burden of depression, can be used to evaluate it ${ }^{10-12}$. 


\section{METHODOLOGY}

The aim of this study was to evaluate the impact of central $\mathrm{n}$. VII paresis in patients after stroke on mimic functions, mental functions and overall quality of life using international clinical scales and objective measurement of mimicry changes using two-dimensional video analysis $^{13-15}$.

The prospective study included 99 patients in the subacute stage of a stroke. Patients were initiated into rehabilitation on the second day after the stroke. Initially they were in the "stroke unit" /ICU/ - Neurology Clinic, Hospital Olomouc - and after transfer rehabilitation continued at the Department of Sports and Exercise Medicine, University Hospital Olomouc (RHB).

At the beginning of rehabilitation, patients were randomly divided into experimental and control groups. The experimental group consisted of 50 persons of whom 26 were men with an average age of 57 years and 24 women, where the average age was 62 years. The control set consisted of 49 persons - 27 men, where the average age was 60 years and 22 women with an average age of 61.5 years. In both groups there was speech therapy once a day focused on the treatment of communication disorders and there were rehabilitation exercises twice a day aimed at reconstruction of damaged physical functions of the trunk, arms and legs. In addition, targeted physiotherapy orofacial treatment took place in the experimental group once a day, including rehabilitation of myofascial structures of the face, breathing rehabilitation and targeted facilitation of mimic functions using the elements of orofacial regulation therapy, which seeks creation of physiological function in the orofacial areas by applying pressure, touch, vibration and traction in specific points on the face of activacting the orofacial muscules ${ }^{16,17}$. Central $\mathrm{n}$. VII paresis does not affect the upper half of the face and that makes

Table 1. Number of patients from experimental and control groups before and after rehabilitation divided into groups according to HBGS.

\begin{tabular}{lcccc}
\hline & E_Before & E_After & C_Before & C_After \\
\hline I. group & 0 & 1 & 0 & 0 \\
II. group & 1 & 34 & 2 & 3 \\
III. gorup & 18 & 10 & 18 & 24 \\
IV. group & 27 & 5 & 24 & 20 \\
V. group & 4 & 0 & 5 & 2 \\
VI. group & 0 & 0 & 0 & 0 \\
\hline
\end{tabular}

the difference in approach to treatment. In "central type" of $\mathrm{n}$. VII paresis the aim of orofacial therapy is to relax spastic muscles, restore selective functions of orofacial muscles and re-educate fundamental mimicy and communication (verbal and non-verbal) features, and functions during food intake.

Clinical evaluation took place at the beginning of therapy and after 4 weeks of therapy. Mimicry was assessed via a House - Brackmann Grading System standardized clinical questionnaire ${ }^{3}$ and via device measurement - twodimensional video analysis - measuring changes in distance of control points on the paretic corner of the mouth and earlobe ${ }^{13,15}$, mental functioning was evaluated according to the Beck Inventory of Depression ${ }^{11}$ and overallselfsufficiency was assessed via questionnaire by Bartel with determination of Bartel Index (BI) (ref. ${ }^{18}$ ) and via the modified Rankin score ${ }^{19}$.

Basic statistics (arithmetic mean, standard deviation) were calculated for individual parameters. The data were statistically verified using single-factor analysis of variance for repeated measures with statistical significance set at $P<0.05$ and as a subsequent post-hoc test the Fischer LSD test was used to compare individual groups in repeated measurements. Further, correlation of mimicry changes (with mental function and the overall state) was tested using the Spearman correlation coefficient at $P<0.001$. For graphical representation we used the average values of monitored parameters and their standard deviation.

\section{RESULTS}

The results show that there is a statistical improvement in mimic functions in both groups. Statistically significant improvement of mimic functions can be observed in the

Table 2. Number of patients from experimental and control groups before and after rehabilitation divided into groups according to modified Rankin score.

\begin{tabular}{lcccc}
\hline & E_Before & E_After & C_Before & C_After \\
\hline I. group & 0 & 1 & 0 & 0 \\
II. group & 4 & 30 & 3 & 19 \\
III. group & 6 & 10 & 4 & 24 \\
IV. group & 33 & 9 & 32 & 6 \\
V. group & 7 & 0 & 10 & 0 \\
VI. group & 0 & 0 & 0 & 0 \\
\hline
\end{tabular}

E_before RHB - number of probands in experimental group before rehabilitation

E_after RHB - number of probands in experimental group after rehabilitation

C_before RHB - number of probands in control group before rehabilitation

C_after RHB - number of probands in control group after rehabilitation

I. group - fully self-sufficient according to modified Rankin scale

II. group - mild helplessness

III. group - slight helplessness

IV. group - medium helplessness

V. group - heavy helplessness

VI. group - death 
experimental group, as the evaluation of results by HBGS (Table 1, Fig. 1) and results using 2D video analysis show - changes in distances between corner of the mouth and earlobe after 4 week treatment (Fig. 2).

Statistically significant improvement of the condition in the experimental group can be seen again in the evaluation of mental functions according to BDI-II (Fig. 3) and the overall quality of life according to BI (Fig. 4) and modified Rankin score (Table 2).

A very close relationship with a positive Spearman correlation coefficient value of $0.69(P<0.001)$ was found in the correlation of changes in mimicry and psychological functions. Comparing mimicry (HBGS) and selfsufficiency according to BI showed a loose relationship with a positive Spearman correlation coefficient value of $0.53(P<0.001)$.

A very close relationship with a positive Spearman correlation coefficient value of $0.72(P<0.001)$ was found between correlation of changes in mimicry (HBGS) and the overall quality of life according to the modified Rankin score.

\section{DISCUSSION}

Our study demonstrates positive results in patients who have undergone targeted orofacial rehabilitation. Significant mimicry improvement of two and more degrees according to HBGS can be seen after 4 weeks in 31 cases (62\%), mimicry improvement by one degree according to HBGS occurred in 18 cases $(36 \%)$. The condition remained the same in 1 case $(2 \%)$, and there was no upset in the experimental group. Unlike the control group, there was spontaneous improvement by two degrees or more in 2 cases (4\%), a slight improvement in one grade occurred in 20 cases $(40.8 \%)$, an unaltered state remained in 28 cases $(55.2 \%)$.

Similar results were reached by Svensson et al. ${ }^{20}$ in their study comparing the effect of mimic EMG biofeedback in 23 patients after stroke to a control group of 12 patients without EMG mimic therapy. In their study, they observed the recovery of facial functions after 1 month in both groups, and there were normal facial functions or only mild dysfunctions after 6 months in two-thirds of patients. Patients with right-sided facial disability had significantly better results than patients with left-sided facial disability.

Furthermore, in their study, Svensson et al. ${ }^{20}$ provide the same functional state of mimicry (according to HBGS), sensitivity and awareness of facial asymmetry in both experimental and control groups 6 months after the inception of stroke. Based on this finding the authors in conclusion did not recommend mimic EMG biofeedback therapy in patients after stroke with n. VII paresis. They predicted spontaneous remission of central n. VII paresis. It should be noted that this study was conducted on a relatively small sample of patients and the results did not reach statistical significance. In comparison with Svensson et al. ${ }^{20}$, our study involved a sufficiently large set of patients with results that are statistically significant and which confirm the positive effects of orofacial physiotherapy with central paresis after stroke.

In assessing depression after stroke using the BDI-II we again find significant improvement in mental functions in the experimental group, with 45 cases (90\%) showing significantly improved mental state, 5 cases (10\%) unchange and in no cases was the condition impaired. In the control group we observed improvement in 29 cases or $60 \%$, in 20 cases $(40 \%)$ the condition remained the same and in case was the condition impaired.

In both groups all patients used antidepressants from the group of SSRI (citalopram $20 \mathrm{mg} /$ day) in the effective dose.

Depression after stroke (post-stroke depression - PSD) occurs in $40-60 \%$ of patients ${ }^{6-9}$. Patients with depression after stroke have a significantly poorer self-sufficiency prognosis, worse rehabilitation effect, worse quality of life, longer hospitalization period, and impaired ability to return to the home environment ${ }^{6,7,10}$. Women have greater difficulty accepting the facial change and orofacial rehabilitation plays a more important role here ${ }^{9,10}$. Mikulik ${ }^{9}$ and Svensson et al. ${ }^{20}$ suggest that in a small number of patients there is spontaneous alteration in mimicry and PSD remission, without treatment. In contrast, studies by Roth et al. ${ }^{6}$ and Rima et al. ${ }^{7}$ are associated with better modification of cognitive function and neurological deficit including central paresis of the facial nerve in patients with PSD treated with antidepressants and psychotherapy.

Assessment of overall self-sufficiency and quality of life using the BI and modified Rankin score shows improvement in both groups. A more significant effect of therapy can be seen in the experimental group. The difference in results is at the border of statistical significance $(P=0.049)$. It seems that mimicry impairment plays a minor role in self-sufficiency and overall quality of life. The same conclusions were reached by other authors, where the most significant negative prognostic factors influencing the self-sufficiency and quality of life of patients after stroke were repeated stroke attacks, advanced age of patients, immobility, urine and stool incontinence, cognitive dysfunction, and impaired ability to communicate ${ }^{21-26}$.

Svensson et al. ${ }^{20}$ reached similar conclusions, where half a year after stroke, they found no significant difference between the experimental and control groups.

Relatively little attention is paid to the issue of mimicry disorders after stroke in the scientific literature. Nevertheless, the consequences of mimicry disorders have repercussions not only at a somatic (orofacial), but also psycho-social level ${ }^{7,10}$. More attention is devoted to peripheral $\mathrm{n}$. VII paresis and to the effects of rehabilitation, where significant positive results are observed in the treatment of mimicry after targeted rehabilitation ${ }^{27,28}$. It should be noted that the etiopathogenesis of central $n$. VII paresis is different from peripheral $n$. VII paresis and thus the orofacial therapy should be modified accordingly. 


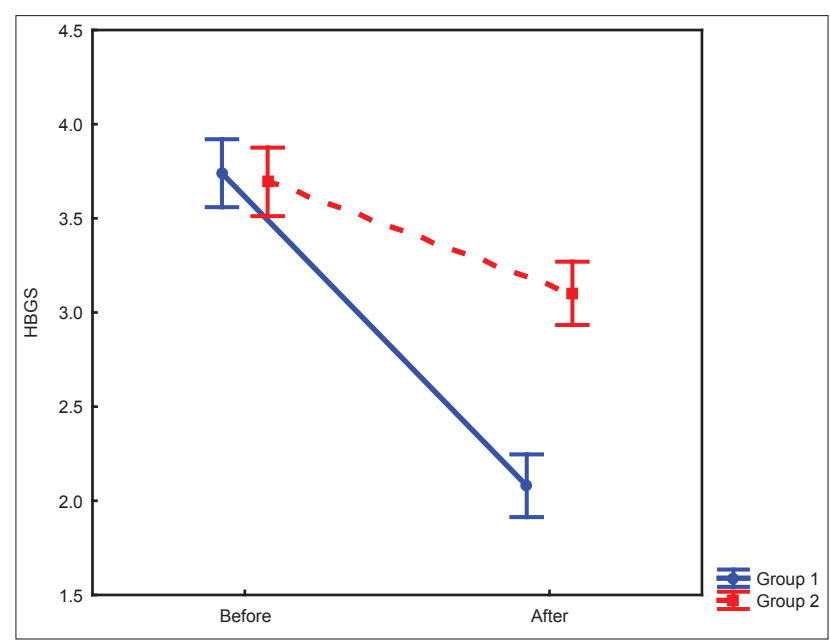

Fig. 1. Change in mimic functions after rehabilitation according to HBGS.

Before - average value of HBGS score before rehabilitation After - average value of HBGS score after rehabilitation HBGS - score values according to HBGS Group 1 - experimental group of patients Group 2 - control group of patients

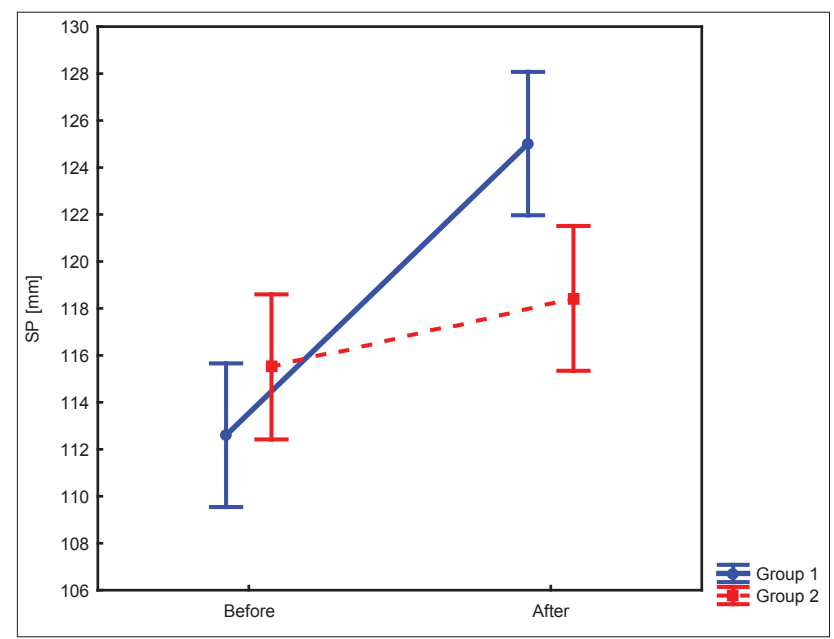

Fig. 2. Average values of change in distances between corner of the mouth and earlobe in $\mathrm{mm}$ assessed at $2 \mathrm{D}$ video analysis at maximal voluntary mouth pouting before and after RHB.

Before - average value of distance between corner of the mouth and earlobe in $\mathrm{mm}$ before rehabilitation

After - average value of distance between corner of the mouth and earlobe in $\mathrm{mm}$ after rehabilitation

$\mathrm{SP}$ - distance between corner of the mouth and earlobe in $\mathrm{mm}$

Group 1 - experimental group of patients

Group 2 - control group of patients

\section{CONCLUSION}

Orofacial rehabilitation as a part of complex rehabilitation care contributes considerably to improving the quality of life after stroke with orofacial function disorder. After complete stabilization of basic life functions and after improving the overall functional state, orofacial rehabilitation is one of the most important aspects of rehabilitation for the patient.

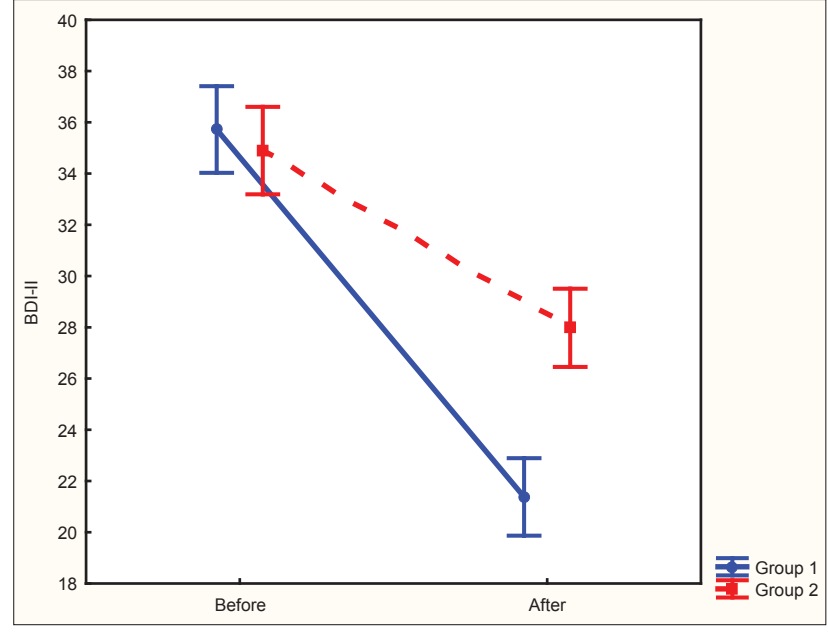

Fig. 3. Evaluation of depression according to BDI-II before and after rehabilitation.

Before - average value of depression score before rehabilitation After - average value of depression score after rehabilitation BDI-II - score values of depression according to BDI-II Group 1 - experimental group of patients Group 2 - control group of patients

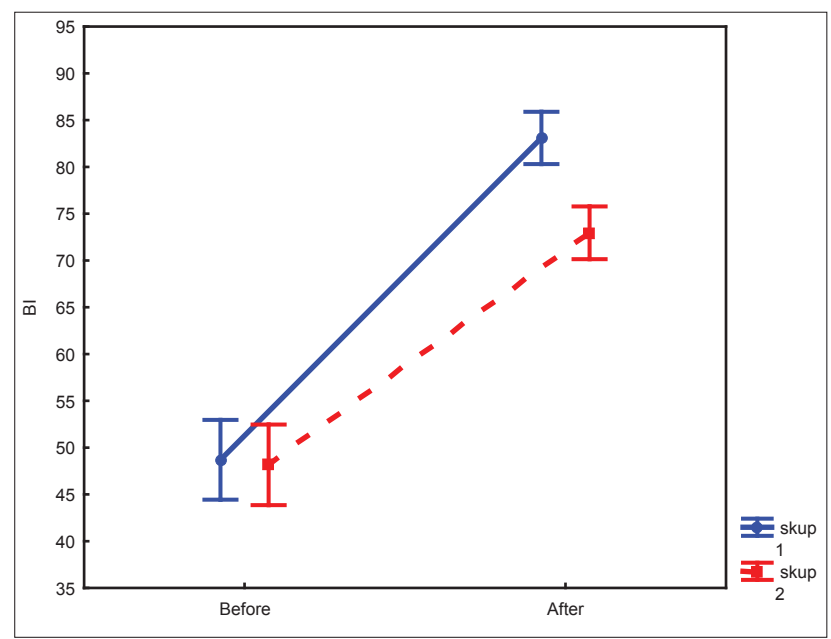

Fig. 4. Evaluation of self-sufficiency according to BI.

Before - average value of BI score before rehabilitation After - average value of BI score after rehabilitation

HBGS - score values according to BI

Group 1 - experimental group of patients

Group 2 - control group of patients

Our study confirms the positive effect in orofacial function improvement after 4 weeks - improvement of mimicry, alleviation of depression and improvement of the overall quality of life in patients after stroke with the central n. VII paresis, who had - in addition to physiotherapy and speech therapy - targeted orofacial therapy. 


\section{REFERENCES}

1. Kalita Z, and all. Acute stroke. Prague: Maxdorf; 2006.

2. Amber Z. Neurology. Prague: Charles' University; 1999.

3. House JW, Brackmann DE. Facial nerve grading system. Otolaryngol Head Neck Surg 1985;93:146-7.

4. Yen TL, Driscoll CL, Lalwani AK. Significance of House-Brackmann facial nerve grading global score in setting of differenial facial nerve function. Otol Neurotol 2003;24:118-22.

5. Reitzen SD, Babb JS, Lalwani AK. Significance and reliability of House-Brackmann Grading System for regional facial nerve function. Otolaryngol Head Neck Surgery 2009;140(2):154-9.

6. Rima MD, Murali R, Aisha Sh, Aparna Sh. Poststroke Depression. Topics in Stroke Rehabilitation 2008;15(1):13-21.

7. Roth J, Preiss M, Uhrova T. Depression in neurology (Czech: Deprese v neurologicke praxi). Prague: Galen; 1999.

8. Bilge C, Kocer E, Kocer A, Türk Börü U. Depression and functional outcome after stroke: effect of antidepressant therapy on functional recovery. Eur J Phys Rehabil Med. 2008;44(1):13-8.

9. Mikulik R. Organic depression in pateients after stroke. In: Depression in neurology. Prague: Galen; 2001. p. 22- 30.

10. Aben I, Verhey F, Lousberg R, Lodder J, Honig A. Validity of The Beck Depression Inventory, Hospital anxiety and Depression scale SCL-90 and Hamilton depression rating scale as instruments for depression in stroke patients. Psychosomatics 2002;43:386-93.

11. Beck AT, Steer AR, Bown GK. The Beck Depression Inventory Second Edition. Manual. San Antonio 1996, TX: The Psychologica Corporation.

12. Dozois DJ, Dobson K, Ahnberg JL. A psychometric evaluation of the Beck Depression Inventory- II. Psychological Assesment 1998;10(2):83-9.

13. Janura M, Zahalka F. Kinematic analysis of movemen of human Universitas Palackianae of Olomouc, 2004.

14. Janura M. Aplication of $3 \mathrm{D}$ videography in the analysis of movement. Acta universitatis Palackianae Gymnica 1998; 28.

15. Carter JA, Pomeroz VM, Richrds J. The feasibility of kinematic measure of lip closure during meaningful speech. Disabil Rehabi 2000;22(18):820-6.
16. Castillo Morales R. Die Orofaziale Regulationstherapie. Munchen, Berlin, Heidelberg: Pflaum; 1998.

17. Konecny P, Elfmark M, Urbanek K. Facial paresis after stroke and its impact on patients' facial movement and mental status. Journal of Rehabilitation Medicine 2011; 43:73-5.

18. GOLDBERG, M.: The internet stroke center. Bartel index. [cited 2009 May 15]. Available from: http://www.strokecenter.org/trials/scales/ barthel.pdf.

19. GOLDBERG, M.: The internet stroke center. Rankin scale. [cited 2009 May 15]. Available from: http://www.strokecenter.org/trials/scales/ rankin.html.

20. Svensson BH, Christiansen LS, Jepsen E. Treatment of central facial nerve paresis with elektromyography biofeedback and taping cheek. A controled clinical trial. Ugeskr Langer 1992;154(50):3593-6.

21. Jongbloed L. Prediction of function after stroke: a critical review. Stroke 1986;17:765-76.

22. Vanaskova E. Krajickova D, Tosnerova V. Rehabilitation after stroke in department of neurology.Ces a Slov Neurol Neurochir 1996;59/92:33-9.

23. Vanaskova E, Tosnerova V, Milacek Z, Waberzinek G, Bukac J. Prediction of clinical tests and stabilography for patients after stroke. Ces a Slov Neurol Neurochir 2002; 69/98:245-49.

24. Heinemann AW, Linacre JM, Wright BD, Hamilton BB, Granger CV. Prediction of rehabilitation outcome with disability measures. Arch Phys Med Rehabil 1994;75:133-43.

25. Pettersen R, Dahl T, Wyller TB. Prediction of long-term functional outcome after stroke rehabilitation. Clin Rehabil 2002;16(2):149-59.

26. Williams LS, Weinberger M, Harris LE, Biller J. Measuring quality of life in a way that is meaningful to stroke patients. Neurology 1999:53(8):1839-43.

27. Cardoso JR, Teixeira EC, Moreira MD, Blue de Oliveira AS. Effects of excercise on Bell's palsy. Otol Neurotol 2008;29:557-60.

28. Ross B, Nedzielski JM, McLean JA. Efficacy of feedback training in long-standing facial nerve paresis. Laryngoscope 1991;101(7):74450 . 